\title{
Global Dissemination of Endoscopic Submucosal Dissection for Early Gastric Cancer
}

\author{
Hajime Isomoto \\ Key words: endscopic submucosal dissection, early gastric cancer, education, training
}

(Inter Med 49: 251-252, 2010)

(DOI: 10.2169/internalmedicine.49.3074)

Gastric cancer is one of the most common cancers. Early detection is critical for its curative treatment. Endoscopic mucosal resection (EMR) is accepted as a treatment of choice for differentiated early gastric cancer (EGC). Recently, Gotoda et al. provided important information on the risks of lymph node metastasis in a large EGC series (1). Based on their analyses, the expanded criteria for endoscopic resection of EGC are proposed: mucosal cancer without ulcer findings irrespective of tumor size; mucosal cancer with ulcer findings $\leq 3 \mathrm{~cm}$ in diameter; and minute $(<500$ $\mu \mathrm{m}$ from the muscularis mucosae) submucosal invasive cancer $\leq 3 \mathrm{~cm}$ in size due to their nominal risks of lymph node metastasis. Nevertheless, the snaring-based procedure of EMR is limited in the lesion size and is not reliable for EGCs with ulcer findings. In fact, elevated EGCs of less than $2 \mathrm{~cm}$ in diameter or small $(\leq 1 \mathrm{~cm})$ depressed EGCs without ulceration is listed in the standard criteria for EMR.

Endoscopic submucosal dissection (ESD) has been developed as a new endoluminal therapeutic technique for superficial gastrointestinal neoplasia (2). After submucosal injection and circumferential incision around the lesion, direct dissection of the submucosa underneath the lesion is performed by using specialized devices including an insulationtipped diathermy knife (2). ESD has the advantage over EMR with respect to curative resection, removing even larger or ulcerated EGC lesions in an en bloc manner $(2,3)$. ESD allows precise histological assessment of the resected one-piece specimens in order to guide further management and to stratify a patient's risk for developing metastasis. As a result, it may prevent residual disease and local recurrence following treatment for $\operatorname{EGCs}(2,4)$. Moreover, long-term outcome of EGC patients treated by ESD can be excellent, a recent study has shown that the 5-year overall and diseasespecific survival rates of EGC patients treated by ESD were $97.1 \%$ and $100 \%$, respectively (4).

Although the prior results of ESD are promising, most studies on clinical outcomes of ESD were conducted at selected and advanced institutes. ESD requires endoscopic skill and sufficient experience and it is associated with higher risks of procedure-related complications including bleeding and perforation $(2,4)$. However, EGC patients who most benefit from ESD are increasingly being offered the option even in low-volume institutions that adapt ESD later, and hence, deal with smaller numbers of EGC patients. Recently, the Japanese health insurance approves ESD for EGC and esophageal cancer lacking the metastatic risks. Therefore, how to disseminate the ESD procedure globally is considered an important task.

In this issue of Internal Medicine, Hotta et al. document a multi-center, one-year, questionnaire survey on the clinical outcomes of ESD for early gastric neoplasm, comparing between 6 high-volume and 24 low-volume ESD centers throughout Nagano prefecture (5). Complete en bloc resection rate (CERR) and the incidence of complications were analyzed in a total of 703 patients with early gastric neoplasm. The CERRs in low- and high-volume centers were both excellent for the lesions that met the standard criteria, being no less than $90 \%$. The CERR in low-volume centers $(82.6 \%)$ was acceptably high even for the expanded criteria lesions, and statistically equivalent to that in high-volume centers $(86.2 \%)$. The procedure-related perforation and bleeding were comparably low, irrespective of the centers' volume. This study has the limitation in terms of the retrospective, questionnaire-based design. Neither detailed data on the clinicopathological characteristics of each patient nor long-term follow-up data is available. Nevertheless, it is of clinical significance to demonstrate that gastric ESD could be performed safely and effectively at low-volume centers. The possible reasons for this feasibility are raised in this paper; the authors have held ESD live demonstration seminars and organized the Nagano ESD Study Group. Video presentations on ESD and experts' lectures at the meetings might 
improve the procedure skills and experience.

In National Cancer Center Hospital, a representative highvolume ESD education center, a trainee is required at least 30 cases to gain proficiency in techniques (6), albeit in the current study (5), the learning curves of the participants were not assessed. Clearly, ESD requires extensive training and its reiteration for archiving proficiency, and idealistically, a trainee is to perform the procedure under close supervision and mentoring of an experienced endoscopist who offers adequate advice on site whenever necessary. If this is not the case, maybe in most low-volume institutions, the harvested porcine gastrointestinal tract organs are inexpensive, and a ready-to-use means to become used to handling the devices. The live porcine model simulates a more realistic setting of ESD, allowing trainees to climb their learning curves in a shorter time (6). For its future success, a rigorous training system for ESD is warranted to be established and standardized. Thus, we believe that the global dissemination of this procedure can be a great boon for much more patients suffering from EGCs and other gastrointestinal neoplasia.

\section{References}

1. Gotoda T, Yanagisawa A, Sasako M, et al. Incidence of lymph node metastasis from early gastric cancer: estimation with a large number of cases at two large centers. Gastric Cancer 3: 219-225, 2003.

2. Gotoda T, Yamamoto H, Soetikno RM. Endoscopic submucosal dissection of early gastric cancer. J Gastroenterol 41: 929-942, 2006.

3. Watanabe K, Ogata S, Kawazoe S, et al. Clinical outcomes of endoscopic mucosal resection for gastric tumors: historical pilot evaluation between endoscopic submucosal dissection and conventional mucosal resection. Gastrointest Endosc 63: 776-782, 2006.

4. Isomoto H, Shikuwa S, Yamaguchi N, et al. Endoscopic submu- cosal dissection for early gastric cancer: a large-scale feasibility study. Gut 58: 331-336, 2009.

5. Hotta K, Oyama T, Akamatsu T, et al. A comparison between high-volume and low-volume centers of outcomes of endoscopic submucosal dissection for early gastric neoplasms: Multi-center retrospective questionnaire study conducted by the Nagano ESD Study Group. Intern Med 49: 253-259, 2010.

6. Gotoda T, Friedland S, Hamanaka H, Soetikno R. A learning curve for advanced endoscopic resection. Gastrointest Endosc 62: 866-867, 2005.

(C) 2010 The Japanese Society of Internal Medicine http://www.naika.or.jp/imindex.html 